\title{
Changes in Sexuality of Castration-Resistant Prostate Cancer Patients under Hormone Therapy in Japan: A Case Study
}

\author{
Yoshiyuki Kashiwagi ${ }^{1}$, Shige Kakinohan ${ }^{2}$ \\ ${ }^{I}$ Graduate School of Health Sciences, University of the Ryukyus, Okinawa, Japan \\ ${ }^{2}$ Graduate School of Health Sciences, University of the Ryukyus, Okinawa, Japan \\ Corresponding author: Yoshiyuki Kashiwagi (newstyle.clubana@gmail.com)
}

\begin{abstract}
Background: Hormone therapy is an important treatment for male patients with prostate cancer. However, it can produce changes in the genitalia and sexuality of the patients. Purpose: This study aimed to investigate the relations between changes in male genitalia and sexuality in the castration-resistant prostate cancer patients. It also proposed concrete interventions to ease pain and fear towards body image changes.

Methods: This study used a descriptive qualitative design. The subjects were two castration-resistant prostate cancer patients undergoing chemotherapy and endocrine therapy. The data were collected through semi-structured interviews and analyzed using verbatim reports and content analysis.

Results: The study indicated that the effects of hormone therapy treatment on the sexuality were largely categorized as: "surprises and anxieties about the shrinkage of male genitalia", "masculinity loss", "difficulty adapting the situation", and "connection with others".

Conclusion: It became clear that the prostate cancer patients had pains not only for prognosis, but also for physical changes, relationship changes with others and sexuality changes through their treatments.
\end{abstract}

Keywords: Prostate cancer; chemotherapy; hormone therapy; sexuality 


\section{BACKGROUND}

In recent years, there is a steady increase of cancer population worldwide. Prostate cancer is the fourth most common cancer in both sexes combined and the second most common cancer in men (IARC, 2015). The total age-standardized incidence rate of prostate cancer is 31.1 per 100.000 . This incidence rate is the second largest for men after lung cancer; the total age-standardized incidence rate of lung cancer is 34.2 per 100.000 (IARC, 2015). With respect to 5 -year survival rate, it is $25.6 \%$ for prostate cancer patients. Compared with that of lung cancer patients $(8.2 \%)$, prostate cancer shows a higher survival rate (IARC, 2015). This would be because there are various treatment modalities including surgery, radiation therapy, hormone therapy and chemotherapy for prostate cancer and also because prostate cancer is a relatively slow-growing cancer (Japan Urological Association, 2012).

Despite the age, family history, and ethnicity have been mentioned as the risk factors of prostate cancer, a widely spread westernized lifestyle (Japan Urological Association, 2012), Prostate-Specific Antigen test (Givannucci, et al., 1993) and aging society are now being focused on having strong relations with the increase in prostate cancer incidence (IARC, 2015). Even in Japan, the incidence rate has been increasing rapidly along with population aging (Nakagawa, et al., 2011), and this should be because aging leads to a longer exposure to male hormones.

In Japan, in addition to multidisciplinary treatments (surgery, chemotherapy, and radiation therapy), hormone therapy is also another popular treatment (Akaza, 2014). This is because, in general, prostate cancer is androgen-dependent and even advanced prostate cancer responds well to androgen deprivation (Nakagawa, et al., 2011) (Nishiyama, 2011). However, a radical cure is not expected for patients with such advanced prostate cancer (Miki, 2006).

There are different kinds of side effects of androgen deprivation treatment, so-called hormone therapy, for prostate cancer and one of the most common side effects is physical changes (e.g., hairless, luster skin, a decline of sexual desire and shrunk male genitalia) (Akaza, 2014). Before medical castration with hormone therapy has begun, surgical castration -removing testicles - was common. However, since a surgical castration tends to hurt male's pride, patients had difficulties with accepting surgery (Ozono, 2014). In addition, although removing adrenal glands was occasionally performed because the invasion was relatively big as surgical castration, it has not widely prevailed, and instead hormone therapy has been mainly provided (Ozono, 2014). Hormone therapy does not cause invasion as big as surgical castration does. However, there are a few physical side effects on male genitalia and it is hard to say that those side effects do not hurt male's pride as much as surgical castration does.

There are quite a few prostate cancer patients who had been taking only hormone therapy at first and started taking chemotherapy after they moved onto castration-resistant prostate cancer (Japan Urological Association, 2012). Then, they eventually reached an advanced stage. Considering this treatment path, sexuality changes due to physical side effects (e.g. shrinking male genitalia) should be recognized not just as physical side effects but as one of the total pains. Moreover, it can be assumed that easing such pain 
can bring positive influences on the condition of castration-resistant prostate cancer patients and also can help them to accept treatments and death.

However, it would be very rare to hear about the shameful side effects related to male genitalia both from patients and medical professionals. Especially in Japan, a subject related to sex and sexuality is regarded as something taboo, and thus only a few medical professionals and patients talk about that (Komatsu, et al., 2001). The side effects of hormone therapy are likely to have some influences on the male sexuality, though (Casey, Corcoran, \& Goldenberg, 2012). In addition, castration-resistant prostate cancer patients have a fear of death and psychological pain towards body image changes. In this situation, the support of nurses is definitely needed (Kakeya, 2007; Shaughnessy, Ireland, Pelentsov, Thomas, \& Esterman, 2013). Traditionally in Japan, it was regarded as a big deal to talk about sex due to the sense of shame or personal values (Yoshihara, 2014). Instead of qualitative studies through direct contact with patients (e.g. interview), there were quite a few quantitative studies with questionnaires (Yoshihara, 2014). Through this study, the relations between changes in male genitalia and sexuality of the castrationresistant prostate cancer patients were clarified, and concrete interventions to ease pain and fear towards body image changes were proposed.

\section{OBJECTIVE}

This study aimed to investigate the relations between changes in male genitalia and sexuality for castration-resistant prostate cancer patients and interventions to ease pain and fear towards body image changes.

\section{METHODS}

This study used a qualitative descriptive design and was carried out at the A hospital with more than 300 beds in Japan. The subjects were 2 castration-resistant prostate cancer patients undergoing chemotherapy on an outpatient basis and endocrine therapy. The regimens were the Docetaxel plus Prednisolone (chemotherapy) combined with Degarelix (endocrine therapy). The survey was conducted in May 2015. The data were collected through semi-structured interviews using guidelines for 2 patients. The survey contents included the thoughts and feelings that the patients had from the first day of their diagnosis until the day of each interview. Specifically, the thoughts and feelings towards undergoing endocrine therapy with medical castration, physical and physiological changes caused by therapy and the effects on their pride and sexuality were asked. Each interview took about one hour in a private room over chemotherapy in consideration of their privacy. Verbatim reports were made every time, and the credibility and stringency of data/analyzed results were confirmed by each patient.

The data were analyzed using verbatim reports which included: (1) using coding process toward each mean based on verbatim reports, (2) discussing and classifying the contents of coding related to the side-effects of symptoms of endocrine therapy, (3) sampling subcategory and category and (4) analyzing using content analysis.

An ethical approval for this study was obtained from the ethics board of the A hospital (Date of approval: 19 January 2015). Before the survey, the participants were given clear explanations of the study protocol, and an informed consent was attained using printed 
instructions and agreement documents. The participants were informed of their rights not to participate and freedom to withdraw at any time from the study. All of the data were kept confidential and anonymous.

\section{RESULTS}

Each case was analyzed by the qualitative inductive approach (Table 1).

\section{Cases}

Patient A was in his early sixties. He had been working as a businessman in Tokyo area since he graduated from high school in Southern Japan till he started chemotherapy. He had a wife and two daughters. At the initial stage, he was diagnosed with bone metastasis and he took a surgery to remove that part. After the surgery, he underwent endocrine therapy. However, he was diagnosed with castration-resistant prostate cancer later on. Since then, he had started taking both endocrine therapy and chemotherapy.

Patient B was in his early seventies. He had been running a company before he opened a Japanese style restaurant after he graduated from high school. Since he started taking the treatments for cancer, his son was in charge of operating the restaurant. Whenever the restaurant was crowded with customers, he sometimes helped in the kitchen. He had a wife and two sons; his younger son got married already. He originally had a chronic renal failure and had been receiving follow-ups. He came to A hospital after pointing out his high PSA scale. A distant metastasis was not yet diagnosed, and therefore he took the endocrine therapy. Later on, he was diagnosed with castration-resistant prostate cancer. Since then, he had started taking both endocrine therapy and chemotherapy.

Table 1. Effects on sexuality of the treatment

\begin{tabular}{ll}
\hline Category & Sub-category \\
\hline Surprises and anxieties about & Surprises toward shrinkage of male genitalia \\
shrinkage of male genitalia & Anxieties about shrinkage of male genitalia \\
& $\begin{array}{l}\text { Pains of not being able to talk about what they } \\
\text { are going through }\end{array}$ \\
& Lack of information \\
& Changes in body images \\
Masculinity loss & Recognition for themselves as a man \\
& Difficulty adapting the physical changes \\
related to side effects & Fear for prognosis \\
Lifulty adapting the situation & Soneliness \\
& Sorry for family \\
\hline
\end{tabular}

\section{Surprises and anxieties about shrinkage of male genitalia}

This category consists of 4 sub categories: 1) surprises toward shrinking male genitalia, 2) anxieties about shrinking male genitalia, 3) pains not to be able to talk about what they are going through, and 4) lack of information.

With respect to surprises toward shrinking male genitalia, patient A said "My testicles have been shrunk", "it was a great shock that my things are getting shrunk to be honest" 
and "I wondered why my things got shrunk". Patient B said "I know there are inevitable side effects with anticancer agents. But I did not expect I would experience any side effects to my male parts".

With respect to anxieties about shrinking male genitalia, patient A said "It is unstoppable since my things are shrunk against my will because of anticancer agent" and "I cannot stop the treatment so I have to accept my non-manly body. Otherwise, cancer is going to develop".

With respect to pains not to be able to talk about what they are going through, patient A said "I have been concerned about my male genitalia. But I cannot talk about my shrunk testicles to my family and even to my doctor". Patient B said "To whom can I talk about my shrunk testicles? It was really harsh not to be able to talk and ask about my concerns related to testicles."

With respect to lack of information, patient A said "It would have been very different if my doctor told me this kind of side effect would happen beforehand" and "My doctor kept telling me that I should undergo cancer therapy and he did not mention much about side effects from that. I could have prepared for what would happen to my testicles if nurses let me know about the side effect before the treatment starts". Patient B said "My doctor told me about the treatment shortly and done. As for the side effects, he just said I should read this book about anticancer agent. For patients, that kind of short and too simple explanation is not enough. Also, the book did not say anything about shrinking testicles. I would not expect medical professionals to tell me everything but patients need more information to prepare for what we are going through. If we know what would happen in advance, we will be able to understand what is going on to our bodies".

\section{Masculinity loss}

This category consists of 2 sub categories: 1) changes in body images, and 2) recognition for themselves as a man.

With respect to changes in body images, patient A said "My skin has been smoother and body hair has been thinner". Patient B said "I am no longer a man. My face is not sharp anymore and my skin has become very smooth. It was sad to see my hair fallen off, however, the saddest things was to see my things that used to be big got shrunk".

With respect to recognition for themselves as a man, patient A said "I doubt if I am a man since my testicles are getting smaller and my body seems feminine. My sex is definitely a male, though. My daughter told me she was jealous since my skin is very smooth and I don't need to shave as a joke. But I am very sad whenever I see my totally changed parts of my body." Patient B said "I am not sure if I am a man or a woman because my body has changed this much. It is sad to pass away with this not manly body, even though I was born as a man." 


\section{Difficulty adapting the situation}

This category consists of 2 sub categories: 1) difficulty adapting the physical changes related to side effects, and 2) fear for prognosis.

With respect to difficulty adapting the physical changes related to side effects, patient A said "There is nothing like adapting. This is because the testicles are automatically getting shrunk against my will. I cannot stop this. I am too ashamed to go to hot springs. Nobody will look at my testicles though." Patient B said "I am working in the food service industry. But I prefer not to serve so I usually help cooking in the kitchen. I used to go to hot springs, but now I cannot go with this shameful body. I have lost one of my favorite things to do for fun."

With respect to fear for prognosis, patient A said "There is no radical cure so I do want to quit this injection once after it became ineffective. When I am in a coffin, I would like to be manlier than now. I am so sorry for my family. I will be gone sometime soon, even after such a long harsh treatment." Patient B said "It would be nice if I can get fully recovered. But even after these treatments along with shocking side effects, cancer will not be removed completely. I know if I stop the treatment, the cancer will keep developing, but it is hard to accept myself, which is way different from what I used to be."

\section{Connection with others}

This category consists 2 sub categories: 1) loneliness, and 2) sorry for family.

With respect to loneliness, patient A said "I am sad and lonely. I cannot live that long and my body has already changed this much." Patient B said "I had fun chatting with my customers. I could forget about my cancer at that moment. But I can no longer be able to serve. I can hear my wife, son and customers are chatting and laughing but I cannot join. I feel so lonely."

With respect to sorry for family, patient A said "My foot got swollen and also I feel pain because the cancer metastasized to the bones. Then I need my family's help to make myself to take a bath. Although my family never said they were surprised when they saw my body, I am shamed to show my changed body and also feel sorry to ask them for help." Patient B said "Since the therapy started, I have lots of changes in my body, such as feeling dull and losing my hair, and I stopped serving. I feel really sorry for my wife and son to burden them. Whenever I tell them I am sorry to be diagnosed with prostate cancer, they respond that I should not feel that way. But I feel so sorry for them to say that."

\section{DISCUSSION}

It has not been clear that how often shrunk male genitalia incident happens as a side effect of degarelix since the injection sites are usually considered as a major concern (Astellas, 2015). Consequently, not many of those symptoms and results related to male genitalia are recognized by the medical professionals. The symptoms and matters related to male genitalia are very sensitive and shameful, therefore, it is very difficult for the patients to tell about their concerns to the medical professionals. Thus, it can be said that any side 
effects related to male genitalia are "unnoticeable" side effects in clinical practices. Through this study, it became clear that the unique and shameful side effects related to male genitalia increased the patients' psychological anxieties and pain. These side effects by GnRH antagonist are inevitable since it prevents the testes from secreting testosterone which develops prostate cancer though.

Neither patient A nor B tried to avoid this side effect; they just believed it would have been much easier to go through the chemotherapy if medical professionals let them know about this side effect in advance. However the reality is that for medical professionals, especially for female nurses, it is very hard to explain the side effect related to male genitalia. Therefore, it can be concluded that it would be one of the best ways for prostate cancer patients to be informed about the side effects by reliable male doctors or nurses beforehand so that they do not have to experience unnecessary anxieties and psychological pain.

In Japan, it seems that subject related to sexuality has tended to be dealt as a taboo and both patients and medical professionals rarely talk about this subject (Komatsu, et al., 2001). On the other hand, a previous study has reported that nurses who have experienced nursing practices related to sexuality have little awareness that they are not good at talking about this subject (Sakai, Mizuno, Hamamoto, \& Sato, 2012). Nurses should understand the side-effects and teach patients about these symptoms, along with hearing the patients' anxieties and other concerns at each treatment. These processes would decrease the patients' anxieties and would help them to adapt. In addition, it is also necessary to tell the patients' family about the side-effects. This is because, like the patients in this study, when the patients need assistance from their family, having knowledge on the side-effects in advance make it easier for the family members to cope with the physical changes on the patients' bodies.

There are few qualitative studies that have dealt with sexuality changes along with shrunk male genitalia. There are quite a few studies that have dealt with sexual dysfunction due to hormone therapy though. This would be because this topic has been thought very sensitive and shameful for both patients and researchers (Yoshihara, 2014). Moreover, it would also be true that cultural and religious factors that make people view sexual topics as taboos hinder the researchers to conduct such studies.

Through this study, it became clear that these two patients had been going through surprises, anxieties, pains and loneliness. Also, it was clear that they had been suffering from psychological and social pains along with physical pains due to sexuality changes in the process of the treatment. These results indicated that nurses should concern about the patients' sexualities and help them ease their various pains. With regards to the patients' cultural and social backgrounds, medical professionals are in need to step into the topics related to sexuality.

Although the WHO proposed that total pains include physical, psychological, social and spiritual pains, this study would like to indicate that sexuality should be included as one of the total pains. This study explained that human beings have both sexual functions and sexual roles, and it is assumed that having changes on those functions and roles could 
cause various pains. This study had explained the sexuality pain and showed that it was related to such other pains as physical, social and psychological pains. Therefore, it can be indicated that the sexuality pain should be a concern and further studies should be carried out to confirm its importance.

As a limit of this study, the targets were only two patients, and therefore it cannot generalize the situation and results. Further studies which involve more participants are required.

\section{CONCLUSION}

In this study, changes in sexuality along with physical changes such as shrunk male genitalia due to the hormone therapy were investigated. As a result, it became clear that the prostate cancer patients had pains not only for prognosis, but also for physical changes, relationship changes with others and sexuality changes through their treatments. For patients who are living in isolated circumstances with such fear, shame, pain and loneliness, the proper interventions by medical professionals are needed to release them from those pains. This study proposed to recognize the sexuality pain as one of the total pains which should not be neglected.

\section{REFERENCES}

Akaza, H. (2014). New era of the treatment of CRPC in Japan. Japanese Journal of Cancer and Chemotherapy, 41(7), 804.

Casey, R. G., Corcoran, N. M., \& Goldenberg, S. L. (2012). Quality of life issues in men undergoing androgen deprivation therapy: a review. Asian Journal of Andrology, 14, 226-231.

Givannucci, E., Rimm, E., Colditz, G., Stampfer, M., Ascherio, A., Chute, C., \& Willett, W. (1993). A prospective study of dietary fat and risk of prostate cancer. Journal of the National Cancer Institute, 85, 1571-1579.

IARC. (2015). Estimated incidence, mortality, and 5-year prevalence: men. Retrieved 11 5, 2015, from GLOBOCAN 2012: Estimated Cancer Incidence, Mortality and Prevalence Worldwide in 2012: http://globocan.iarc.fr/Pages/fact_sheets_population.aspx

IARC. (2015). Prostate Cancer. Retrieved 11 05, 2015, from GLOBOCAN 2012: Estimated incidence, mortality and prevalence worldwide in 2012: http://globocan.iarc.fr/Pages/fact_sheets_cancer.aspx

Japan Urological Association. (2012). The Japanese guidelines for prostate cancer. (L. a. Ministry of Health, Editor) Retrieved 10 13, 2015, from Medical Information Network Distribution Service: http://minds.jcqhc.or.jp/n/med/4/med0032/G0000435/0042

Kakeya, J. (2007). Present status of the functions and subjective burdens of urination/defecation/sex in patients with prostate cancer -evaluation of support for outpatients-. The Bulletin of Niimi College, 28, 119-123.

Komatsu, H., Nomura, M., Okamitsu, K., Ito, E., Suzuki, K., \& Minamikawa, M. (2001). Effects of aging and chronic illness on sexuality of older adults. Journal of St. Luke's Society for Nursing Research, 5(1), 41-50. 
Lindley-Jones, M., \& Finlayson, B. J. (2000b). Triage nurse requested x rays-are they worthwhile? Journal of Accident \& Emergency Medicine, 17(2), 103-107. doi: 10.1136/emj.17.2.103

Mackway-Jones, K., Marsden, J., \& Windle, J. (Eds.). (2006). Emergency triageManchester Triage Group (2 ed.). Oxford: Blackwell Publishing Ltd.

Miki, T. (2006). Treatment for cancer specifically diagnosed in high-aged population, prostate cancer. Japanese journal of geriatrics, 43, 71-73.

Minister of Health The Republic of Indonesia. (2009). The standard of emergency departments in the hospitals. (856/Menkes/SK/IX/2009). Jakarta: Ministry of Health The Republic of Indonesia.

Ministry of Health The Republic of Indonesia. (1999). Material of PPGD training series: emergency care system and national policy (1 ed.). Jakarta: Ministry of Health The Republic of Indonesia.

Nakagawa, T., Hiraoka, Y., Kitou, K., Nagai, A., Manabe, A., Hashimoto, T., . . S Sutou, Y. (2011). Study of effectiveness of cooling injection site for pain relief when injecting LH-RH analogues into prostate cancer patients. Japanese Journal of Pharmaceutical Health Care and Sciences, 37(3), 157-163.

Nishiyama, T. (2011). Paradigm shift in the concept of hormonal milieu of prostate cancer. Niigata medical journal, 125(11), 581-601.

Nixon, V. (2008). Identification of the development needs for the emergency care nursing workforce. International Emergency Nursing, 16(1), 14-22. doi: 10.1016/j.ienj.2007.10.001

Ozono, S. (2014). Role of urologists in the era of new treatment modalities for CRPC in Japan. Japanese Journal of Cancer and Chemotherapy, 41(7), 823-826.

Quattrini, V., \& Swan, B. A. (2011). Evaluating care in ED fast tracks. Journal of Emergency Nursing, 37(1), 40-46. doi: 10.1016/j.jen.2009.10.016

Sakai, A., Mizuno, M., Hamamoto, M., \& Sato, S. (2012). Sexuality as an aspect of nursing care for prostate cancer patients and the awareness of nurses providing such care. Journal of japan of Society of Nursing Research, 35(4), 57-64.

Seiger, N., van Veen, M., Steyerberg, E. W., Ruige, M., van Meurs, A. H. J., \& Moll, H. A. (2011). Undertriage in the Manchester triage system: an assessment of severity and options for improvement. Archives of Disease in Childhood, 96(7), 653-657. doi: 10.1136/adc.2010.206797

Shaughnessy, P. K., Ireland, C., Pelentsov, L., Thomas, L. A., \& Esterman, A. J. (2013). Impaired sexual function and prostate cancer: a mixed method investigation into the experiences of men and their partners. Journal of Clinical Nursing, 22, 34923502.

Shields, L., \& Hartati, L. E. (2003). Nursing and health care in Indonesia. Journal of Advanced Nursing, 44(2), 209.

Woolwich, C. (2000). Accident and emergency: theory into practice. London: Balliliere Tindall.

Yoshihara, S. (2014). A literature review of excretion and sexuality in prostate cancer. Journal of The Showa University Society, 74(6), 654-660.

Yoshihara, S. (2015). The experiences of patients with advanced prostate cancer. Journal the Japanese Red Cross Society of Nursing Science, 15(1), 31-38. 